\title{
Intraventricular haemorrhage in the preterm infant without hyaline membrane disease
}

\author{
J. S. WIGGLESWORTH, P. A. DAVIES, I. H. KEITH, AND S. A. SLADE
}

From the Department of Paediatrics and Neonatal Medicine, Institute of Child Health, Hammersmith Hospital, London

SUMMARY The clinicopathological associations of 33 singleton infants who died with intraventricular haemorrhage (IVH) without hyaline membrane disease (HMD) ('IVH only') were compared with those of 39 infants who died with IVH + HMD over the same gestation range in order to determine what factors other than those related to HMD may contribute to the pathogenesis of IVH. The incidence of 'IVH only' was inversely related to gestational age in the Hammersmith birth population, whereas the incidence of IVH + HMD rose to a peak at 28-29 weeks' gestation. Infants with 'IVH only' lived longer on average than those with IVH + HMD despite a lower birthweight and shorter gestation. Infants who died in the first 12 hours from 'IVH only' had suffered severe birth asphyxia but in those who died later the main symptom was recurrent apnoea. Fewer infants with 'IVH only' were given alkali therapy or were connected to the ventilator as compared to those with IVH+HMD, but there were no differences in alkali therapy in those who lived for 12 hours or more. In the 'IVH only' group there was a high incidence of haemorrhage from other sites and of bacterial infections.

It is suggested that, in the absence of HMD, extreme immaturity is the main factor determining the occurrence of IVH. Birth asphyxia, apnoeic attacks, haemorrhage, and infections may play subsidiary roles, possibly through development of metabolic acidosis.

Recent papers on intraventricular haemorrhage (IVH) have stressed its association with hyaline membrane disease (HMD) in $50-95 \%$ of cases (Harcke et al., 1972; Leech and Kohnen, 1974; Anderson et al., 1976). The frequency of this association has led workers to consider IVH as a complication of HMD and to ignore the group of infants who die with IVH alone.

We have previously shown that infants who died with IVH without HMD at Hammersmith Hospital (1966-73) were of significantly lower birthweight and lower gestational age than those who died with IVH + HMD, and have discussed evidence that intravenous alkali administration may have contributed to the incidence of IVH in the latter group (Wigglesworth, 1976; Wigglesworth et al., 1976). We have now undertaken a more detailed study of the clinicopathological associations of the infants who developed IVH without HMD in order to determine what factors, other than those associated with HMD, may contribute to the pathogenesis of IVH.

Received 5 October 1976

\section{Materials and methods}

The study is based on the 111 singleton cases of IVH associated with germinal layer haemorrhage which came to necropsy at Hammersmith Hospital over the years 1966-73. Details of the background population of admissions to the neonatal unit and the methods of study have been given previously (Wigglesworth et al., 1976). Infants with serious malformations or severe Rhesus isoimmunization have been excluded.

Within the group there were 34 infants with IVH without HMD. As all but one were below 32 weeks' gestation, we compared these 33 cases with those cases of IVH +HMD which were within the same gestational age range (39 out of a total of 77).

\section{Results}

Birthweight and gestational age. The infants with IVH only were significantly smaller and less mature than those with IVH +HMD $(P<0.001$ for both 
Table 1 Clinical findings

\begin{tabular}{|c|c|c|c|}
\hline & IVH only (33) & $I V H+H M D(39)$ & $P$ \\
\hline $\begin{array}{l}\text { Maternal age (mean } \pm \text { SEM) } \\
\text { Pre-eclampsia } \\
\text { Primigravidae } \\
\text { Length of labour (h) (mean } \pm \text { SEM) } \\
\text { Abnormal delivery } \\
\text { Caesarean section } \\
\text { Gestation (w) (mean } \pm \text { SEM) } \\
\text { Birthweight (g) (mean } \pm \text { SEM) } \\
\text { Sex ratio } M / F \\
\text { Intubation at birth } \\
\text { Mechanical ventilation } \\
\text { Fits } \\
\text { Hypoglycaemia } \\
\text { Intravenous alkali } \\
\text { Lowest recorded body temperature }\left({ }^{\circ} \mathrm{C}\right) \text { (mean } \pm \text { SEM) }\end{array}$ & $\begin{array}{l}26 \cdot 4 \pm 1 \cdot 0 \\
1(3 \%) \\
22(67 \%) \\
9 \cdot 78 \pm 2 \cdot 00 \\
11(34 \%) \\
1(3 \%) \\
26 \cdot 9 \pm 0 \cdot 31 \\
990 \pm 38 \\
1 \cdot 2 \\
18(55 \%) \\
15(45 \%) \\
14(42 \%) \\
8(24 \%) \\
23(70 \%) \\
33 \cdot 2 \pm 0 \cdot 23\end{array}$ & $\begin{array}{l}25 \cdot 8 \pm 1 \cdot 1 \\
5(13 \%) \\
17(44 \%) \\
6 \cdot 23 \pm 1 \cdot 28 \\
12(31 \%) \\
6(15 \%) \\
28 \cdot 6 \pm 0 \cdot 28 \\
1270 \pm 41 \\
1 \cdot 05 \\
19(49 \%) \\
31(79 \%) \\
12(31 \%) \\
12(31 \%) \\
37(95 \%) \\
33 \cdot 7 \pm 0 \cdot 15\end{array}$ & $\begin{array}{l}\text { NS } \\
\text { NS } \\
0.042 \\
0.033 \\
\text { NS } \\
0.084(\mathrm{NS}) \\
<0.001 \\
<0.001 \\
\mathrm{NS} \\
\mathrm{NS} \\
<0.01 \\
\mathrm{NS} \\
\mathrm{NS} \\
<0.025 \\
0.082 \text { (NS) }\end{array}$ \\
\hline
\end{tabular}

birthweight and gestational age differences) despite limiting the comparison to infants of less than 32 weeks' gestation (Table 1). The frequency of death with IVH only in the Hammersmith Hospital singleton liveborn population was inversely related to gestational age (Fig. 1). In contrast, IVH + HMD in the same population rose to a peak incidence at 28-29 weeks' gestation (Fig. 1).

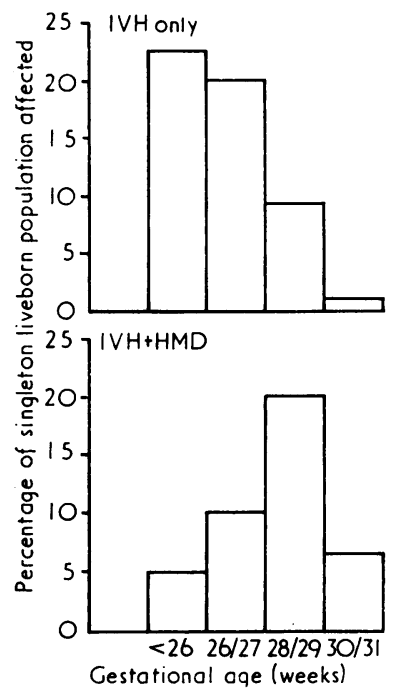

Fig. 1 Incidence of IVH only and IVH+HMD in singleton live births at different gestational ages below 32 weeks-Hammersmith Hospital births 1966-73. Addition of upper and lower columns gives total IVH incidence in each gestational age group.

Age at death. There was a significantly different distribution of age at death between the groups (Fig. 2). Infants with HMD+IVH died most frequently during the second 24 hours of life (mean

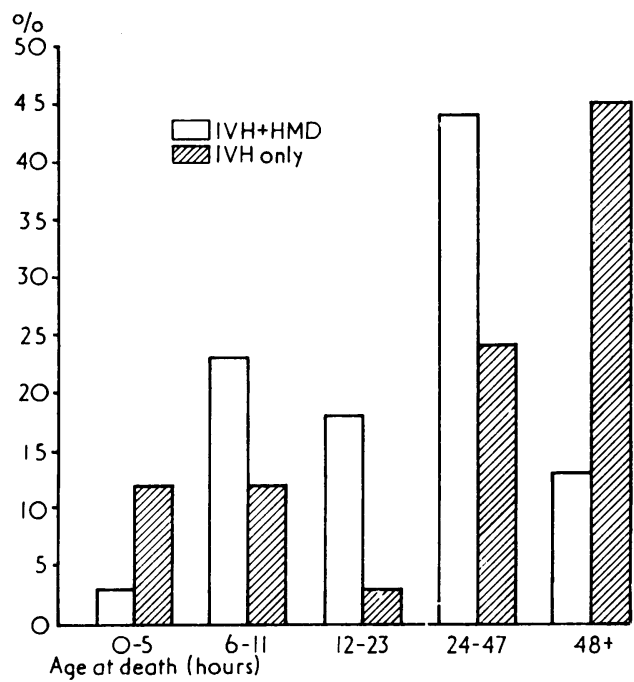

Fig. 2 Percentage of infants with IVH only and with IVH $+H M D$ dying at different ages (gestational ages below 32 weeks). Distributions are significantly different $\left(\chi^{2}=11 \cdot 76, P<0 \cdot 01\right.$, on $4 \times 2$ table with deaths below 12 hours age amalgamated).

age at death $27 \cdot 9 \pm 3 \cdot 3 \mathrm{~h}$ ), whereas those with IVH only either died within the first 12 hours of life or survived until the second day or later (mean age at death $47 \cdot 2 \pm 6 \cdot 9 h^{*}$ ). Nearly half of them lived for $\mathbf{4 8}$ hours or more and one survived for over a week. Early deaths were more frequent in the infants born at Hammersmith but only 3 of 16 died in the first 6 hours.

Clinical associations. Factors analysed are shown in Table 1. Fewer infants with IVH only were connected to the ventilator or given intravenous alkali therapy as compared to the IVH + HMD

*Excluding the infant who survived for over a week. 
infants. There were marginally more primigravid mothers and longer labours in the 'IVH only' group. Other maternal and infant factors including maternal age, incidence of pre-eclampsia, incidence of abnormal delivery (forceps, breech, or caesarean section), incidence of fits, incidence of hypoglycaemia, and the lowest recorded body temperature showed no difference between the groups.

Details of alkali therapy are given in Table 2.

Table 2 Alkali therapy (in cases surviving 12 hours or more)

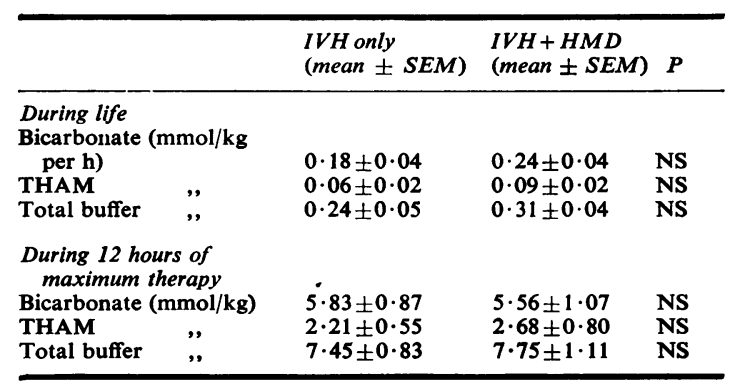

Although fewer babies with IVH only were given alkali therapy there was no significant difference between the groups in any aspect of alkali therapy when infants who lived for less than 12 hours are excluded.

Clinical presentation of 'IVH only' group. We carefully examined the notes of the infants who died with IVH without HMD to see whether there were common features in their clinical presentation and to see why alkali therapy had been used in such a high proportion of a group of babies who did not have HMD at necropsy. It became clear that there were several modes of presentation.

(a) Severe birth asphyxia. 5 infants who died in the first 12 hours had severe birth asphyxia and remained in poor condition throughout their few hours of extrauterine life.

(b) Respiratory distress presumed due to HMD. This was diagnosed in 12 infants. In retrospect the symptoms were mild in relation to the extreme immaturity of some of these babies. Hypoxia and acidosis developed only after apnoeic attacks began. 6 of these infants had massive pulmonary haemorrhage or pneumonia at necropsy.

(c) Recurrent apnoea. 10 infants developed apnoeic attacks within the first 24 hours after birth in the absence of signs of respiratory distress.

(d) Late collapse. 6 infants were well after birth until development of severe apnoea at 24 hours age or later.
Pathological associations. Two additional forms of pathology were detected during life or at necropsy in 19 of 33 infants with IVH only. These were extracranial haemorrhage in 14 cases and bacterial infections in 9 (Table 3). In contrast, only 11 of

Table 3 Additional pathology seen in infants of ' $I V H$ only' group.

\begin{tabular}{|c|c|c|c|}
\hline \multicolumn{2}{|c|}{ Extracranial haemorrhage (14 cases) } & \multicolumn{2}{|l|}{ Bacterial infection (9 cases) } \\
\hline $\begin{array}{l}\text { Extensive bruising } \\
\text { Massive pulmonary }\end{array}$ & 6 & Pneumonia & $5^{*}$ \\
\hline haemorrhage & $5^{*}$ & Positive blood cultures & $6+$ \\
\hline Umbilical cord & 2 & E. coli & 2 \\
\hline Injection or venepuncture sites & 2 & Ps. aeruginosa & 2 \\
\hline Nasopharynx & 2 & Klebsiella sp. & 1 \\
\hline \multicolumn{4}{|l|}{ Ruptured subcapsular } \\
\hline haematoma of liver & $1^{*}$ & $\begin{array}{l}\text { B-haemolytic } \\
\text { streptococcus } \\
\text { Group B }\end{array}$ & 1 \\
\hline
\end{tabular}

* Necropsy finding. + During life or immediately after death.

39 infants with IVH + HMD had either complication (haemorrhage 6, infection 5). The differences between the groups are significant if the total incidence of these complications is considered $\left(x^{2}=5 \cdot 19, \mathbf{P}<0.025\right)$ and if the incidence of haemorrhage is considered separately $\left(x^{2}=5 \cdot 24\right.$, $\mathbf{P}<0.025$ ), but not if infection is considered alone.

Of the 'IVH only' infants with extracranial haemorrhage, 5 were treated by exchange transfusions for a profound fall in haemoglobin level, whereas none of the IVH + HMD group was transfused. Coagulation tests were not performed consistently over the period of study but disseminated intravascular coagulation was suspected clinically in 2 cases. The diagnosis was supported by a platelet count of $15000 / \mathrm{mm}^{3}$ $\left(15 \times 10^{9} / 1\right)$ in one. Both of these infants, and 3 others, had massive pulmonary haemorrhage (Table 3).

Most of the bacterial infections were due to Gram-negative organisms, as indicated by 5 of the 6 positive blood cultures obtained during life or immediately after death (Table 3).

Gestation over 32 weeks. The one infant with IVH only in this group had a birthweight of $2100 \mathrm{~g}$ at 36 weeks' gestation. The infant developed apnoeic attacks from 6 hours, was thought clinically to have HMD, and died at 23 hours age. This case has been excluded from the statistical comparisons and tables.

\section{Discussion}

Our study confirms that there are marked clinical differences between groups of infants dying with 
IVH according to whether they also have HMD. These differences persist despite limiting the comparison to infants of a similar gestational age range. The shorter gestation and lower birthweight of the group with IVH only* as compared to those with additional HMD would readily be explained if they had died very soon after birth, before HMD could develop. Since these infants lived longer on average than those with HMD, the differences cannot be accounted for so simply. The rapid fall in incidence of IVH only with increasing gestation that we observed in the Hammersmith births suggests that in the absence of HMD immaturity is the most important factor determining whether IVH develops or not.

Our infants with IVH only died usually at over 24 hours age, though a few died in the first 12 hours. Fedrick and Butler (1970) reported a similar pattern for their 'pure IVH' group, albeit with a larger proportion $(43.8 \%)$ of deaths in the first 6 hours of life. Increased use of resuscitation measures at birth may have reduced the frequency of early death from IVH alone, either by preventing severe birth asphyxia or by ensuring survival of any infants with surfactant deficiency until structural evidence of HMD developed.

While it is easy to relate the early group of deaths to the effects of birth asphyxia in very immature infants, the late deaths demand a different explanation. The relatively late age at death of some infants with uncomplicated IVH has been noted previously (Amiel, 1964; Fedrick and Butler, 1970). In such cases it is usually not possible to determine whether the initial germinal layer haemorrhage (GLH) develops at several days of age or whether the late collapse is due to rupture of a GLH that has been present since soon after birth. A further possibility is that in the absence of HMD an infant may survive for a longer period after development of IVH.

The higher incidence of primiparous mothers in the group with IVH only agrees with the findings of Fedrick and Butler (1970) for IVH cases as a whole. The slightly longer labours noted in the cases with IVH only would be consistent with the larger number of primigravidae but also with the lack of delivery by caesarean section. It seems unlikely that the differences in length of labour would be sufficient to allow maturation of surfactant pathways in the lungs of one group rather than the other.

*Since our 'IVH only' group includes all infants with IVH asseciated with germinal layer haemorrhage except those with HMD, it is not directly comparable to the 'pure IVH' groups of previous studies (e.g. Amiel, 1964; Fedrick and Butler, 1970) which excluded infants with other forms of additional pathology.
The main feature of infants who died early from IVH only was severe birth asphyxia. Among the rest, despite their relatively prolonged survival, few were symptom free in the first 24 hours. Nearly half were diagnosed during life as having HMD, but in retrospect the main symptom was recurrent apnoea. Recessions, grunting, and tachypnoea were mild or transient. The significance of bleeding problems in these infants is uncertain. Damage to vessels in the germinal layer might be a result of shock associated with the haemorrhage. Alternatively, if the mechanisms we have postulated previously for initiation of IVH are correct (Hambleton and Wigglesworth, 1976; Wigglesworth et al., 1976), the use of transfusions for treating bleeding episodes might act as a precipitating factor by raising the circulating blood volume and altering pressure-flow relationships in the cerebral circulation. Disseminated intravascular coagulation was confirmed only in one case. The possible relevance of this condition. to IVH has been extensively discussed elsewhere (Chessells and Wigglesworth, 1972; Hambleton and Wigglesworth, 1976). The significance of bacterial infection in the infants with IVH only is somewhat doubtful as this form of complication may simply reflect the longer survival seen in this group. Invasion of the bloodstream by Gram-negative bowel organisms might also in some cases be a terminal event in a moribund infant.

Although alkaline buffer therapy was given to fewer of the infants with IVH alone than to those with IVH + HMD the doses administered to infants who survived for 12 hours or more did not differ between the groups. If, as we have argued previously, alkaline buffer therapy plays some role in the high incidence of IVH in babies with HMD it may also have contributed to the incidence of IVH in the babies without HMD. The effect may be relatively small, however, since the doses of alkali given to infants below 30 weeks' gestational age, who constitute the bulk of those with IVH alone, have been lower in all groups than the doses given to infants of greater maturity (Wigglesworth et al., 1976).

The differences between the various groups of babies described in this paper are largely explicable in terms of an increasing severity of stress required to elicit IVH with increasing maturity. Uncomplicated IVH is a condition related primarily to extreme immaturity, but one that can be affected by a number of factors. Severe birth asphyxia in such immature infants is associated with rapid development of, and death from, the condition. At a few hours or days of age recurrent apnoea, haemorrhage, or possibly infection may be similarly 
associated. Each of these conditions could cause IVH through development of some common form of stress such as metabolic acidosis. The more mature infant will only develop IVH if HMD is superimposed, and particularly if exposed to intensive alkaline buffer therapy (Wigglesworth et al., 1976). It should be possible by improved management to reduce considerably the incidence of IVH in infants above 30 weeks' gestation, though the condition is likely to remain a problem in the very immature.

The analyses of our perinatal data bank on which this work is based were performed with the aid of a grant from the Small Grants Section of the DHSS.

\section{References}

Amiel, C. (1964). Hémorragies cérébrale intra-ventriculaires chez le prématuré. II. Les éléments du diagnostic clinique. Biologia Neonatorum, 7, 57-75.

Anderson, J. M., Bain, A. D., Brown, J. K., Cockburn, F., Forfar, J. O., Machin, G. A., and Turner, T. L. (1976). Hyaline-membrane disease, alkaline buffer treatment, and cerebral intraventricular haemorrhage. Lancet, 1, 117-119.
Chessells, J. M., and Wigglesworth, J. S. (1972). Coagulation studies in preterm infants with respiratory distress and intracranial haemorrhage. Archives of Disease in Childhood, 47, 564-570.

Fedrick, J., and Butler, N. R. (1970). Certain causes of neonatal death. II. Intraventricular haemorrhage. Biologia Neonatorum, 15, 257-290.

Hambleton, G., and Wigglesworth, J. S. (1976). Origin of intraventricular haemorrhage in the preterm infant. Archives of Disease in Childhood, 51, 651-659.

Harcke, H. T., Naeye, R. L., Storch, A., and Blanc, W. A. (1972). Perinatal cerebral intraventricular hemorrhage. Journal of Pediatrics, 80, 37-42.

Leech, R. W., and Kohnen, P. (1974). Subependymal and intraventricular hemorrhages in the newborn. American Journal of Pathology, 77, 465-476.

Wigglesworth, J. S. (1976). Buffer therapy and intraventricular haemorrhage. Lancet, 1, 249.

Wigglesworth, J. S., Keith, I. H., Girling, D. J., and Slade, S. A. (1976). Hyaline membrane disease, alkali, and intraventricular haemorrhage. Archives of Disease in Childhood, 51, 755-762.

Correspondence to Dr. J. S. Wigglesworth, Department of Paediatrics and Neonatal Medicine, Institute of Child Health, Hammersmith Hospital, Du Cane Road, London W12 0HS. 